\title{
Synthetic MUC1 breast cancer vaccine containing a Toll-like receptor 7 agonist exerts antitumor effects
}

\author{
YU LIU $^{1,2^{*}}$, LI TANG $^{1,3^{*}}$, NINGNING GAO ${ }^{1}$, YUWEN DIAO $^{1}$, JINGJING ZHONG $^{1}$, \\ YONGQIANG DENG ${ }^{4}$, ZHULIN WANG ${ }^{1}$, GUANGYI JIN ${ }^{1}$ and XIAODONG WANG ${ }^{1}$
}

${ }^{1}$ International Cancer Center, National-Regional Engineering Lab for Synthetic Biology of Medicine, School of Pharmaceutical Sciences, Shenzhen University Health Science Center, Shenzhen, Guangdong 518055; ${ }^{2}$ Department of Research and Education, The Third Affiliated Hospital of Shenzhen University, Shenzhen, Guangdong 518001; ${ }^{3}$ College of Physics and

Optoelectronic Engineering, Key Laboratory of Optoelectronic Devices and Systems of The Ministry of Education and Guangdong Province, Shenzhen University, Shenzhen, Guangdong 518060; ${ }^{4}$ Department of Oral and Maxillofacial Surgery, Shenzhen University General Hospital, Shenzhen University Health Science Center, Shenzhen, Guangdong 518055, P.R. China

Received January 14, 2020; Accepted May 27, 2020

DOI: 10.3892/ol.2020.11762

\begin{abstract}
Adjuvant immunotherapy has recently emerged as a potential treatment strategy for breast cancer. The tumor-associated protein mucin 1 (MUC1) has received increasing attention due to its high expression in numerous types of common tumors, in which MUC1 acts as a cancer antigen. However, the simple mixed composition of an adjuvant and a peptide is not a sufficient rationale for a MUC1 peptide-based vaccine. The present study developed a novel Toll-like receptor 7 (TLR7) agonist-conjugated MUC1 peptide vaccine (T7-MUC1), which elicited an effective immune response and a robust antitumor effect in a mouse breast cancer model. In vitro, T7-MUC1 significantly increased the release of cytokines in mouse bone marrow dendritic cells and spleen lymphocytes, and induced the dendritic cell-cytokine-induced killer response against tumor cells with high MUC1 expression. In vivo, it was observed that the 4T1 tumor weights in mice immunized with the T7-MUC1 conjugate were reduced by $\geq 70 \%$ compared with those in the control group. Furthermore, the therapeutic responses in vivo were attributed to the increase in specific humoral and cellular
\end{abstract}

Correspondence to: Professor Xiaodong Wang or Professor Guangyi Jin, International Cancer Center, National-Regional Engineering Lab for Synthetic Biology of Medicine, School of Pharmaceutical Sciences, Shenzhen University Health Science Center, 1066 Xueyuan Avenue, Shenzhen, Guangdong 518055, P.R. China

E-mail: wangxiaodong@szu.edu.cn

E-mail: gyjin@szu.edu.cn

${ }^{*}$ Contributed equally

Key words: breast cancer, Toll-like receptor 7 agonist, mucin 1, immunotherapy, peptide-based vaccine immunity, including high antibody titers, antibody-dependent cell-mediated cytotoxicity and cytotoxic T-lymphocyte activity. The percentages of $\mathrm{CD}^{+} / \mathrm{CD}^{+} \mathrm{T}$-cells were significantly higher in the T7-MUC1 treatment group compared with those in the control group. Therefore, the results of the present study suggested that the T7-MUC1 vaccine inhibited tumor growth in mice and thus may have potential as a therapeutic candidate in clinical trials for breast cancer immunotherapy.

\section{Introduction}

Mucin 1 (MUC1) is a membrane-associated glycoprotein involved in the protection of mucous membranes and modulation of immune system (1). MUC1 is upregulated in $>70 \%$ of all types of cancer and has important roles in tumorigenicity and tumor progression; thus, this antigen may be a potential target for cancer immunotherapy (2). T-cell epitopes of the MUC1 core domain bind major histocompatibility complex (MHC) class I molecules in their truncated hypoglycosylated form, which directs the MHC-restricted recognition of epitopes $(3,4)$. MUC1-based clinical trials are currently ongoing, including retargeting human $\mathrm{CD}^{+}$and $\mathrm{CD} 4^{+} \mathrm{T}$ cells to tumor-associated MUC1 glycoforms $(5,6)$. In addition, TG4010, a viral vaccine developed by Transgene SA, expresses full-length MUC1 and interleukin (IL)-2, and has demonstrated an association between overall survival and vaccine-induced T-cell responses (7). Lakshminarayanan et al (8) have also constructed a tumor vaccine by covalent attachment of a T-helper epitope and an aberrantly glycosylated MUC1 peptide, which induces immunoglobulin (Ig) $\mathrm{G}$ antibodies and cytotoxic $\mathrm{T}$ lymphocytes (CTLs) against MUC1. However, a lack of immune recognition, resulting from the weak immunogenicity of tumor antigens, is the primary reason for the inadequacy of vaccines to induce potent immune responses (8).

Toll-like receptors (TLRs) are a family of integral membrane proteins that are primarily localized on immune cells, such as dendritic cells (DCs) and macrophages (9). TLRs recognize molecules that are widely shared by 
pathogens, known as pathogen-associated molecular patterns (9). Following the recruitment of adapter proteins, TLR activation leads to the stimulation of myeloid differentiation primary response 88 -dependent signaling and the subsequent release of inflammatory cytokines and stimulatory molecules (10). As a bridge between innate and adaptive immunity, TLRs have been used for conjugation with ligands and antigens, and have been demonstrated to exhibit advantages over non-coupled antigens (11). Among the TLRs, only TLR7 recognizes small synthetic molecules, including nucleoside analogues, which are easier to obtain and modify compared with other biomacromolecules, such as TLR4 and TLR9 ligands (12). TLR7 agonists (T7s) have attracted attention for their promotion of not only antigen-presenting cells (APCs), but also T cells and natural killer $(\mathrm{NK})$ cells $(13,14)$. Our previous study investigated small molecule TLR7 ligands, and a series of T7s were synthesized with significant immunostimulatory activity (15). Our previous study also constructed tumor vaccines by conjugating the $\mathrm{T} 7$ with gastric cancer antigens, which had synergistic antitumor effects with chemotherapeutic agents via T-cell activation and myeloid-derived suppressor cell inhibition (16).

The present study conjugated a novel T7 and MUC1 peptide together (T7-MUC1) for use as a vaccine and examined its immune responses and anti-tumor effects. It was hypothesized that systemic administration of T7-MUC1 may induce antitumor immune responses and elicit an antitumor effect in a mouse breast cancer model by enhancing CTL activity and antibody-dependent cell-mediated cytotoxicity (ADCC). In addition, it was speculated that the therapeutic effect of T7-MUC1 may occur due to non-specific anti-tumor responses elicited by the adjuvant $\mathrm{T} 7$, and specific cellular and humoral immune responses elicited by the MUC1 peptide.

\section{Materials and methods}

Mice and cell lines. 4T1 mouse breast cancer cells, MCF-7 human breast cancer cells, MB231 human breast cancer cells and K562 human leukemia cells (American Type Culture Collection) were cultured in RPMI-1640 medium (K562 cells) or DMEM (4T1, MCF-7 and MB231 cells) (both HyClone; Cytiva), supplemented with 10\% FBS (HyClone; Cytiva), $100 \mu \mathrm{g} / \mathrm{ml}$ penicillin and $100 \mu \mathrm{g} / \mathrm{ml}$ streptomycin (Gibco; Thermo Fisher Scientific, Inc.) at $37^{\circ} \mathrm{C}$ in a humidified atmosphere with $5 \% \mathrm{CO}_{2}$. All experiments were performed with mycoplasma-free cells.

Female 4-week-old BALB/c mice ( $\mathrm{n}=150$; weight, 15-20 g) were purchased from the Medical Laboratory Animal Centre of Guangdong Province. All mice were housed in constant specific pathogen-free laboratory conditions at $18-22^{\circ} \mathrm{C}$ and $50-60 \%$ humidity with a $12 \mathrm{~h}$ light/dark cycle and ad libitum access to water and food. The protocols of the animal experiments were approved by the Laboratory Animal Ethics Committee of Shenzhen University (approval no. AEWC-201712025).

Synthesis of T7 and T7-MUC1. The MUC1 peptide used in the present study is a well-documented murine MUC1 epitope (8). T7 (SZU101), MUC1 and T7-MUC1 were synthesized in our laboratory; MUC1 and T7-MUC1 were synthesized by solid phase using an Fmoc strategy as previously described $(12,17)$. T7-K was prepared separately as an amino acid for T7-MUC1, by first mixing the $\mathrm{T} 7$ activated ester with lysine, and subsequently stirring for $4 \mathrm{~h}$ at room temperature. The structure of T7 was determined as previously described (17).

Cytokine assay. Bone marrow dendritic cells (BMDCs) were generated from the femurs and tibiae of one BALB/c mouse as previously described (12), and were cultured in X-vivo 15 medium (Lonza Group AC) with granulocyte-macrophage colony-stimulating factor (GM-CSF) and IL-4 for 6 days. Spleen lymphocytes were isolated from the same BALB/c mouse used to generate BMDCs as previously described (12) using Mouse Lymphocyte Separation Medium (Dakewe Biotech Co., Ltd.) and cultured in RPMI-1640 medium with $10 \%$ FBS. Cells were seeded in 96-well plates at a density of $5 \times 10^{4}$ cells per well. T7-MUC1 was added at a final concentration ranging between 0.1 and $100 \mu \mathrm{g} / \mathrm{ml}$ and incubated at $37^{\circ} \mathrm{C}$ for $24 \mathrm{~h}$; T7 or MUC1 were used with an equal molar quantity to T7-MUC1. Then, culture supernatants were collected, and the levels of tumor necrosis factor (TNF)- $\alpha$, interferon (IFN) $-\gamma$ and IL-12 were assessed using mouse TNF- $\alpha$ (cat. no. 88-7324-22), IFN- $\gamma$ (cat. no. 88-7314-22) and IL-12 p70 (cat. no. 88-7121-22) ELISA kits (Invitrogen; Thermo Fisher Scientific, Inc.) according to the manufacturer's instructions.

Immunizations and antibody detection. All experiments were routinely performed in groups of eight mice. On days 0 , 14 and 28, the mice were intraperitoneally (i.p.) administered PBS, $20 \mu \mathrm{g}$ MUC1 peptide, $8.5 \mu \mathrm{g}$ T7 or $28 \mu \mathrm{g}$ T7-MUC1. A total of $0.2 \mathrm{ml}$ blood was collected from the tail of the mice 7 days after the last vaccination, and serum was harvested from the blood for serologic assays. Anti-MUC1 IgG, IgG1, IgG2a and $\operatorname{IgM}$ antibody titers were determined by ELISA as previously described (18). ELISA plates (Thermo Fisher Scientific, Inc.) were coated with the MUC1 peptide, and serial dilutions of the serum were allowed to bind to immobilized MUC1. Detection was performed by adding alkaline phosphatase-conjugated anti-mouse antibodies and phosphorylated nitrophenyl phosphate (Sigma-Aldrich; Merck KGaA), and the optical density was measured at $405 \mathrm{~nm}$ using a microplate reader.

Western blotting. Whole cell protein was extracted from K562, MCF-7 and MB231 cells using RIPA lysis buffer with $1 \mathrm{mM}$ PMSF (Beyotime Institute of Biotechnology), and protein concentrations were detected using a bicinchoninic acid assay (Beyotime Institute of Biotechnology). Proteins were separated by $10 \%$ SDS-PAGE and transferred onto PVDF membranes. The membranes were first blocked in 5\% bovine serum albumin (Beyotime Institute of Biotechnology) at room temperature for $1 \mathrm{~h}$, and then incubated with rabbit anti-human MUC1 (cat. no. 14161) or $\beta$-actin (cat. no. 4970) monoclonal antibodies (1:1,000; Cell Signaling Technology, Inc.) at $4^{\circ} \mathrm{C}$ overnight, and finally with goat anti-rabbit peroxidase conjugated-secondary antibodies (cat. no. 7074; 1:2,000; Cell Signaling Technology, Inc.) at room temperature for $1 \mathrm{~h}$. Antibodies bound to the blots were detected using the Clarity Western ECL Substrate (Bio-Rad Laboratories, Inc.). 
DC-cytokine-induced killer cell (CIK) co-culture. Ethics approval was obtained for the use of human tissues in the present study by the Medical Ethics Committee of the Third Affiliated Hospital of Shenzhen University (approval no. 2019-SZLH-LW-009), and informed consent was provided by the healthy donors. PBMCs (peripheral blood mononuclear cells) were isolated from human blood samples of 3 male healthy donors (age range, 25-35 years; mean age, 30 years), and separated by density gradient centrifugation at $400 \mathrm{x} g$ for $30 \mathrm{~min}$, according to the manufacturer's instructions (Tianjin Haoyang Biological Co., Ltd.). DCs and CIKs were cultured from PBMCs as previously described (19). For the induction of CIKs, non-adherent cells were cultured in RPMI-1640 medium containing $1,000 \mathrm{U} / \mathrm{ml}$ IFN- $\gamma, 1,000 \mathrm{U} / \mathrm{ml} \mathrm{IL}-2$ and $100 \mathrm{ng} / \mathrm{ml}$ $\mathrm{CD} 3$ antibody (PeproTech, Inc.) at $37^{\circ} \mathrm{C}$ for 7 days. Adherent cells were differentiated to DCs using 1,000 U/ml GM-CSF, $500 \mathrm{U} / \mathrm{ml} \mathrm{IL}-4$ and $500 \mathrm{U} / \mathrm{ml} \mathrm{TNF}-\alpha$ (PeproTech, Inc.) for 7 days; MUC1 and the T7-MUC1 conjugate were added as an antigen on day 3 or 5. DCs and CIKs were co-cultured between days 7 and 14 with a supplement of 1,000 U/ml IL-2. The phenotype molecules of DCs $\left(\mathrm{CD} 80^{+} / \mathrm{CD}^{2} 3^{+} / \mathrm{CD}^{2} 6^{+}\right)$ and CIKs $\left(\mathrm{CD}^{+} / \mathrm{CD}^{2} 6^{+}\right)$were determined via flow cytometry using a FACScalibur flow cytometer (BD Biosciences) and the FlowJo v10 software (BD Biosciences). The mouse anti-human CD80-FITC (cat. no. 305205), CD83-APC (cat. no. 305311), CD86-PerCP/Cy5.5 (cat. no. 374215), CD3-FITC (cat. no. 300305) and CD56-APC (cat. no. 362503) monoclonal antibodies (1:100) for flow cytometry were purchased from BioLegend, Inc. On day 14, tumor cells (MCF-7, MB231 and K562) and DC-CIKs were used as target cells and effector cells, respectively, at an effector/target cell ratio of 10:1 for $4 \mathrm{~h}$. The levels of lactate dehydrogenase (LDH) released by target cells in the supernatant were determined using a CytoTox 96 Non-Radioactive Cytotoxicity assay (Promega Corporation) according to the manufacturer's instructions.

Evaluation of antitumor effects in vaccinated mice. On day 21 , mice were subcutaneously injected in the mid-back region with $100 \mu \mathrm{l}$ 4T1 cell suspension $\left(1 \times 10^{6}\right.$ cells $/ \mathrm{ml}$ in PBS). The tumor dimensions were measured twice a week, and tumor volume was calculated according to the equation Volume $=\mathrm{LxW}^{2} / 2$, where $\mathrm{L}$ is length $(\mathrm{mm})$ of the long axis of the tumor. On day 42, the mice were sacrificed by $\mathrm{CO}_{2}$ inhalation to minimize animal suffering, and the tumors were surgically dissected, weighed and measured. Additionally, another 30 mice were used for the evaluation of the long-term survival until the mice died naturally or the tumor diameter reached $15 \mathrm{~mm}$.

Hematoxylin and eosin $(H \& E)$ staining. The tumor tissues were cut into thin slices and stained with a Hematoxylin and Eosin Staining kit, according to the manufacturer's protocol (Beyotime Institute of Biotechnology) and observed under a light microscope at x200 magnification.

CTL assay. At the time of sacrifice, the spleens were removed and lymphocytes were obtained by filtering the organs through a sterile nylon mesh $(70 \mu \mathrm{m})$. Then, $4 \mathrm{~T} 1$ tumor cells used as target cells and lymphocytes used as effect cells were seeded in 96-well culture plates (Corning, Inc.) at an effector/target cell ratio of $50: 1$ at $37^{\circ} \mathrm{C}$ for $4 \mathrm{~h}$. The levels of $\mathrm{LDH}$ released by the target cells in the supernatants were determined as aforementioned.

Determination of ADCCs. 4T1 tumor cells (5,000 cells/well) were incubated with serum (1:20 dilution) obtained from the vaccinated mice for $30 \mathrm{~min}$ at $37^{\circ} \mathrm{C}$. NK cells separated from the spleen were used as effectors and were seeded with the antibody-labeled tumor cells in 96-well culture plates (Corning, Inc.) at an effector/target cell ratio of $50: 1$ at $37^{\circ} \mathrm{C}$ for $4 \mathrm{~h}$. The levels of $\mathrm{LDH}$ in the supernatants were determined as aforementioned.

Flow cytometry. At the time of sacrifice, the mouse spleen was collected, and splenocytes were prepared by removing the red blood cells (RBCs) with RBC lysis buffer (BioLegend, Inc.) after separating the cells by a $70-\mu \mathrm{m}$ cell strainer. Subsequently, $1 \times 10^{6}$ cells were stained with the corresponding florescence antibodies and analyzed using a FACScalibur flow cytometer (BD Biosciences) and the FlowJo v10 software (BD Biosciences). The rat anti-mouse CD4-FITC (cat. no. 100406), CD8-PE (cat. no. 100708) and CD3-APC (cat. no. 100236) monoclonal antibodies (1:100 dilution) for flow cytometry were purchased from BioLegend, Inc.

Statistical analysis. Data are presented as the mean \pm SEM from the indicated number of independently performed experiments. Two-way ANOVA with Bonferroni post hoc test was used to compare the tumor volumes in different groups collected over all time points. One-way ANOVA with Bonferroni post hoc test was used for the determination of statistical significance for all other experiments. $\mathrm{P}<0.05$ was considered to indicate a statistically significant difference.

\section{Results}

Synthesis of the T7-MUC1 conjugate. Structures of all of the vaccines used in this study are presented in Fig. 1. T7 (Fig. S1A) was synthesized in the laboratory following a previously described procedure (17), with the ${ }^{1} \mathrm{H}$ and ${ }^{13} \mathrm{C}$ NMR being consistent with the assigned structure (Fig. S2A and B). The certificates of the T7-MUC1 conjugate are presented in Fig. S3A and B, and the ratio of the T7 conjugate to MUC1 was 3:1 (Fig. S1B).

In vitro cytokine release in response to the T7-MUC1 conjugate. In order to evaluate the immunological activity of T7-MUC1, BMDCs and spleen-derived lymphocytes were incubated with different concentrations of T7, MUC1 and T7-MUC1, and ELISA was used to determine the release of cytokines. The results indicated that the levels of IL-12 and TNF- $\alpha$ remained unchanged when BMDCs were incubated with MUC1 alone (Fig. 2A and B). However, the levels of IL-12 and TNF- $\alpha$ in the T7-MUC1 and T7 groups were significantly higher compared with those in the control group. The results also demonstrated that the levels of IL-12 and TNF- $\alpha$ released by BMDCs in the T7-MUC1 group were significantly increased compared those in with the T7 group. A similar trend was observed for the release of IL-12 and IFN- $\gamma$ in spleen lymphocytes (Fig. 2C and D). 


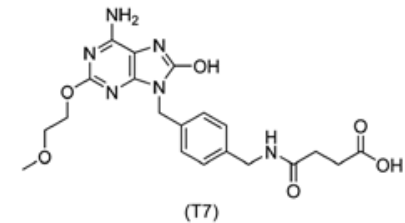

KKKCKLFAVWKITYKDTGTSAPDTRPAP (MUC1)

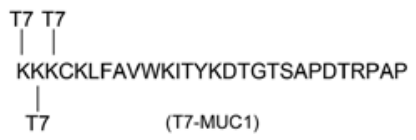

Figure 1. Chemical structures of the synthetic vaccines.

T7-MUC1 conjugate induces Ig G and IgM antibody responses. The anti-MUC1 IgG, IgG1, IgG2a and IgM antibody titers were determined by ELISA. The results demonstrated that $\mathrm{T} 7$ and MUC1 alone had no effect on $\mathrm{IgG}$ and $\mathrm{IgM}$ antibody responses, but the T7-MUC1 conjugate significantly elicited the IgG total, IgG1, IgG2a and IgM antibody responses (Table I).

In vitro cytolytic response in the high MUC1 protein-expressing cells is induced by the T7-MUCl conjugate. To observe the antigen effect of the T7-MUC1 conjugate, the present study developed a DC-CIK co-culture procedure using human samples. DCs and CIKs were cultured separately, and the T7-MUC1 conjugate was added as an antigen on day 3 and 5 to the DC culture. Then, DCs and CIKs were co-cultured on day 7, and the CTL effect was measured on day 14. Western blotting results for the three types of human tumor cells, MCF-7, MB231 and K562, demonstrated high protein expression levels of MUC1 in MCF-7 cells, whereas K562 and MB231 cells exhibited relatively low MUC1 expression; MB231 cells expressed undetectable levels of MUC1 protein (Fig. 3A). The CTL results demonstrated that the conjugate-induced CTLs exerted a more potent effect on MCF-7, but not K562 and MB231 cells (Fig. 3B-D). Therefore, it was speculated that the conjugate-induced CTLs exerted increased target cell cytotoxicity, and this effect was partially dependent on the expression of MUC1 in the target cells.

Antitumor effect of the T7-MUC1 conjugate in a mouse breast cancer model. To assess the antitumor effect of the T7-MUC1 conjugate, BALB/c mice were injected subcutaneously with 4T1 mouse breast cancer cells after two immunizations. Then, 21 days after the 4T1 cell injection, the mice were sacrificed, and the tumors were dissected, weighed and measured. The results demonstrated a significant growth inhibition in the $\mathrm{T} 7$, MUC1 and conjugate-treated groups compared with that in the control group ( $\mathrm{P}<0.05$; Fig. 4A, B and E). Of note, compared with the PBS control, tumor weight was lower (43.3\%) after treatment with T7-MUC1-conjugate than with T7 (63.2\%) or MUC1 (73.7\%) alone (Fig. 4B). The results also demonstrated that the T7-MUC1 conjugate group exhibited a significantly increased long-term survival rate compared with that in the other groups during a 50-day observation period (Fig. 4C). Tumor tissues were also examined by H\&E staining, and it was identified that the T7-MUC1 conjugate exerted an antitumor effect in the mouse breast cancer model (Fig. 4D).

T7-MUC1 conjugate induces tumor-specific immune responses. To determine the capacity of the T7-MUC1 vaccine to elicit a tumor-specific immune response, lymphocytes were isolated from the spleen in the different groups, and the percentages of $\mathrm{CD}^{+} / \mathrm{CD}^{+}$and $\mathrm{CD}^{+} / \mathrm{CD}^{+} \mathrm{T}$ cells were
Table I. ELISA anti-MUC1 antibody titers after three immunizations with T7-MUC1.

\begin{tabular}{lrrrr}
\hline Treatment & IgG total & IgM & IgG1 & IgG2a \\
\hline PBS & 0 & 0 & 0 & 0 \\
T7 & 55 & 13 & 8 & 15 \\
MUC1 & 1,719 & 303 & 920 & 688 \\
T7-MUC1 & 11,147 & 9,552 & 12,890 & 13,257 \\
\hline
\end{tabular}

Titers were defined as the highest dilution yielding an optical density of $\geq 0.1$ relative to normal control mouse sera. MUC1, mucin 1 ; T7, Toll-like receptor 7 agonist; Ig, immunoglobulin.

measured by flow cytometry. No changes in the percentages of $\mathrm{CD}^{+} / \mathrm{CD}^{+} \mathrm{T}$ cells were observed among the groups (Fig. 5A). However, the percentages of $\mathrm{CD}^{+} / \mathrm{CD}^{+} \mathrm{T}$ cells were significantly higher in the T7-MUC1 group compared with in the PBS control group (Fig. 5B). Representative flow cytometry results of $\mathrm{CD}^{+} / \mathrm{CD}^{+} \mathrm{T}$-cells are demonstrated for the PBS control (Fig. 5E) and T7-MUC1 conjugate (Fig. 5F) groups. Further representative flow cytometry results are presented in Fig. S4. Regarding ADCCs and CTLs, the cytotoxicity effects in 4T1 cells induced by NK cells following the addition of antisera or by lymphocytes in the T7-MUC1 group were significantly higher compared with those in the other groups (Fig. 5C and D).

\section{Discussion}

Breast cancer is a major cause of cancer mortality among women, especially in the least developed countries, and the risk factors include reproductive and endocrine dysfunction, obesity and physical inactivity (20). Traditional treatments of breast cancer include surgery, chemotherapy, radiotherapy and endocrinotherapy (20). Currently, immunotherapy is a promising novel therapeutic strategy due to its advantages such as effective targeting and limited side effects (21). The immune system can recognize, process and present tumor antigens, leading to the cross-priming and activation of $\mathrm{T}$ cells and tumor elimination (21). In addition, multiple tumor-associated antigens, such as human epidermal growth factor receptor and MUC1, are targets of novel drugs developed for breast cancer vaccines, a number of which have been demonstrated to be clinically beneficial (22).

In recent years, multimodal cancer vaccines have received increased attention due to their ability to simultaneously stimulate different aspects of the immune system (23). However, it has been revealed to be difficult to obtain vaccines with both cellular and humoral immune responses against MUC1, although either of these responses are associated with a satisfactory outcome in clinical cancer treatment $(24,25)$. It has also been demonstrated that patients with breast cancer who are vaccinated with a 30-amino acid sequence of MUC1 peptide conjugated with keyhole limpet hemocyanin (KLH) and the immune adjuvant QS-21 produce high IgG and IgM antibody titers to MUC1 and high response to KLH, but the T-cell response against MUC1 peptide is minimal 

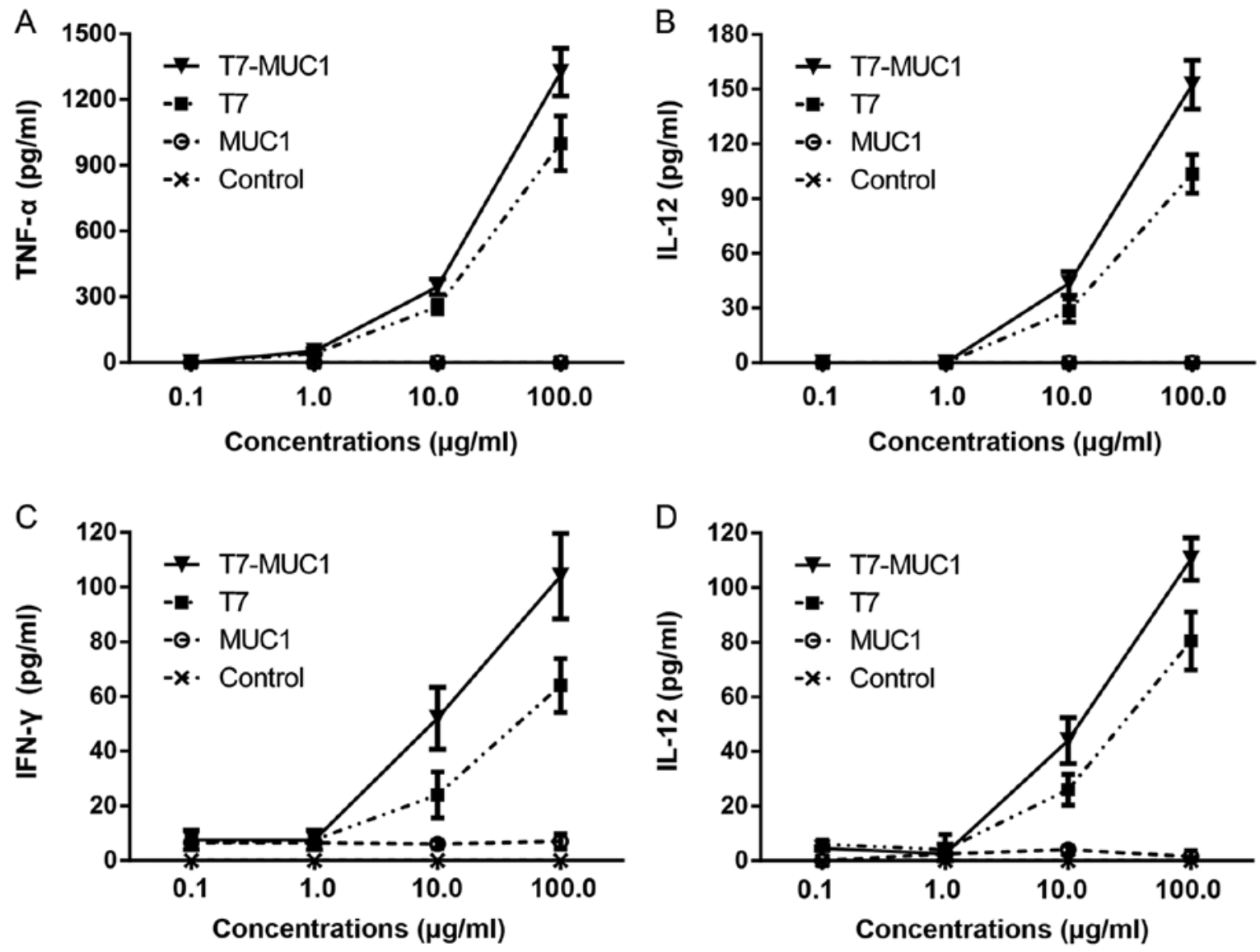

Figure 2. In vitro cytokine release in response to the T7-MUC1 conjugate. (A-D) Induction of (A) TNF- $\alpha$ and (B) IL-12 from BMDCs, and (C) IFN- $\gamma$ and (D) IL-12 from spleen lymphocytes after $24 \mathrm{~h}$ incubation with each treatment. Data are presented the mean $\pm \mathrm{SD} ; \mathrm{n}=3$. IL, interleukin; TNF, tumor necrosis factor; IFN, interferon; BMDCs, bone marrow dendritic cells; MUC1, mucin 1; T7, Toll-like receptor 7 agonist.

A

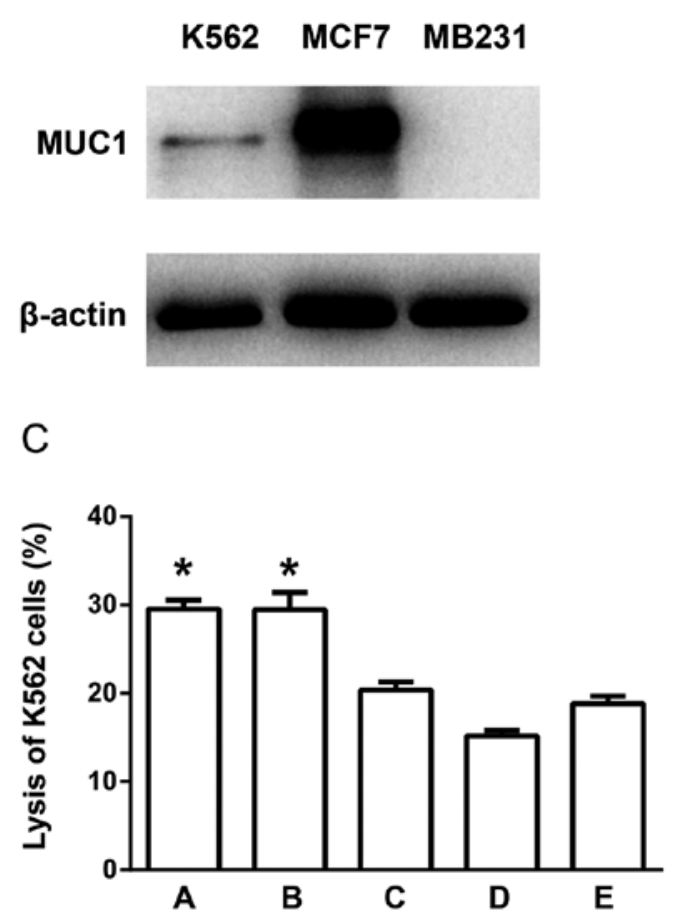

B

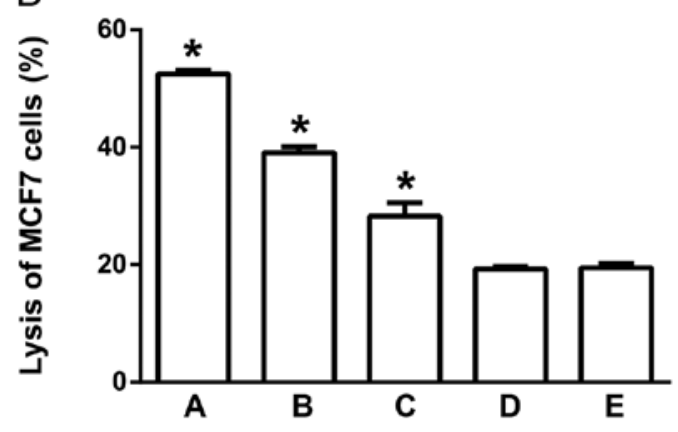

D

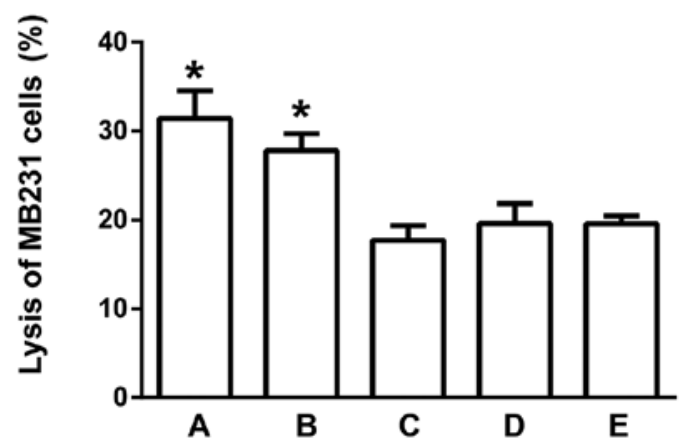

Figure 3. T7-MUC1 conjugate induces a cytolytic response in cells expressing high levels of MUC1 protein. (A) MUC1 expression in MCF-7, MB231 and K562 cells. (B-D) Induction of the CTL effect on (B) MCF-7, (C) K562 and (D) MB231 cells. The groups are as follows: A, T7-MUC1 conjugate added on day 3; B, MUC1 added on day 3; C, T7-MUC1-conjugate added on day 5; D, MUC1 added on day 5; and E, control with no added antigen. Data are presented as the mean $\pm \mathrm{SD} ; \mathrm{n}=5$. "P<0.05 vs. E. MUC1, mucin 1; T7, Toll-like receptor 7 agonist; PBMCs, peripheral blood mononuclear cells; CTL, cytotoxic T lymphocytes. 

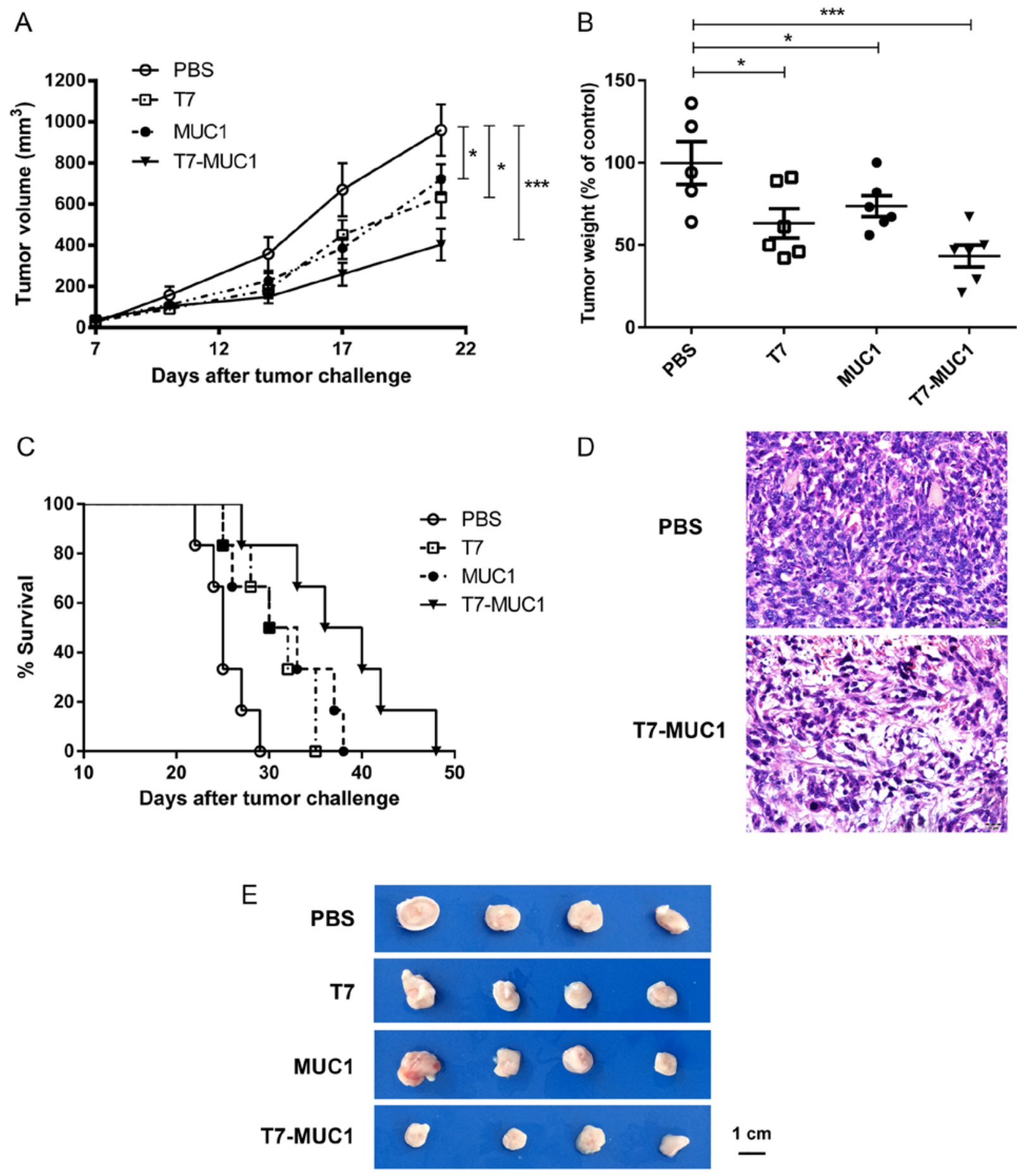

Figure 4. T7-MUC1-conjugate inhibits 4T1 mouse breast tumor growth after two immunizations. (A) Tumor growth curves of tumor volumes measured twice a week until day 21 after tumor implantation. (B) Tumor weights were determined at the time of sacrifice; the mean tumor weight in the PBS control group was $0.75 \pm 0.10 \mathrm{~g}$. (C) Survival curves of the tumor-bearing mice. (D) Representative H\&E staining results of tumor tissues for the PBS control and T7-MUC1 groups. (E) Representative images of the excised tumors from all groups. Data are presented the mean $\pm \mathrm{SD} ; \mathrm{n} \geq 5$. ${ }^{*} \mathrm{P}<0.05$, ${ }^{* * * *} \mathrm{P}<0.001$ vs. $\mathrm{PBS}$. H\&E, hematoxylin and eosin; MUC1, mucin 1; T7, Toll-like receptor 7 agonist.

and inconsistent $(26,27)$. Furthermore, a previous study has reported that lack of glycosylation in MUC1 peptides used in vaccines leads to ineffective immune responses (22). In the present study, following conjugation with $\mathrm{T} 7$, the MUC1 peptide without the glycosylated structure generated an overall therapeutic response, suggesting that the conjugation of T7 and MUC1 may be a potential effective vaccine against breast tumors.
TLR ligands are widely used as adjuvants in the design of vaccines to boost the immunogenicity of antigens, producing strong and long-lasting immunity. For example, TLR2 peptide-overexpressing Bacille Calmette-Guérin triggers MHC II-dependent antigen presentation and elicits $\mathrm{T}$ helper (Th) 1 cytokine release in vitro, leading to increased expansion of the effector and central memory $\mathrm{T}$ cells in vivo (28). Furthermore, Maynard et al (29) have reported that $\mathrm{CpG}$, a 
A
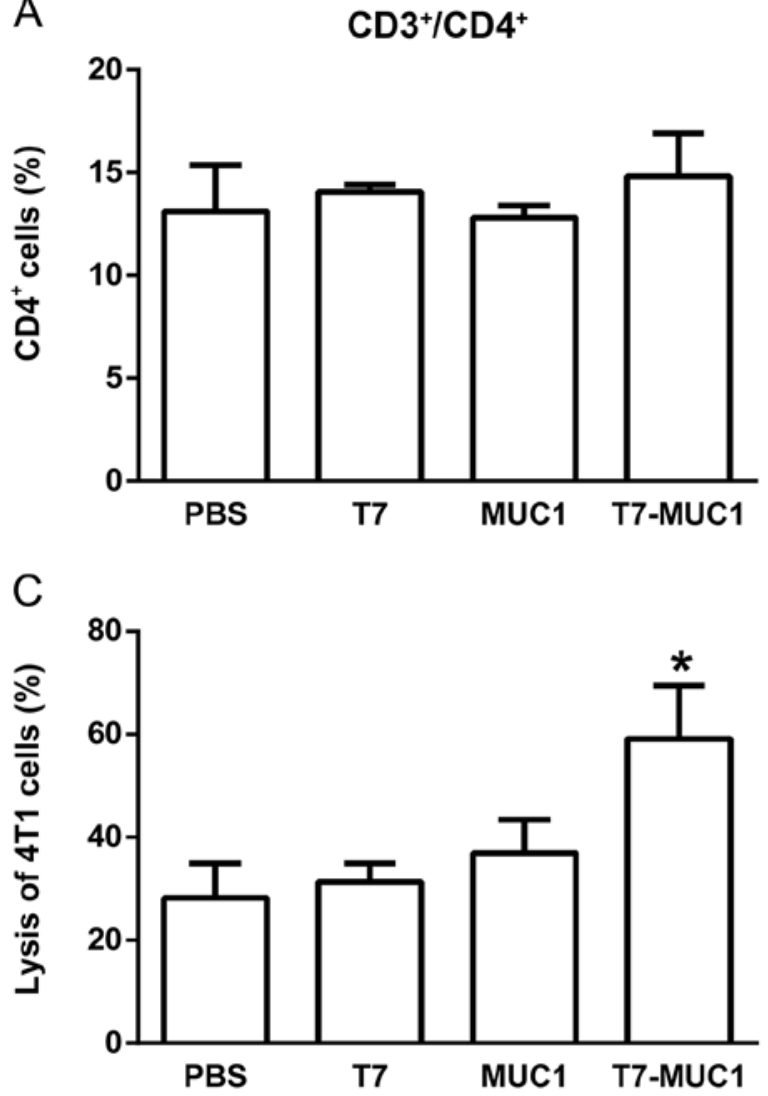

E

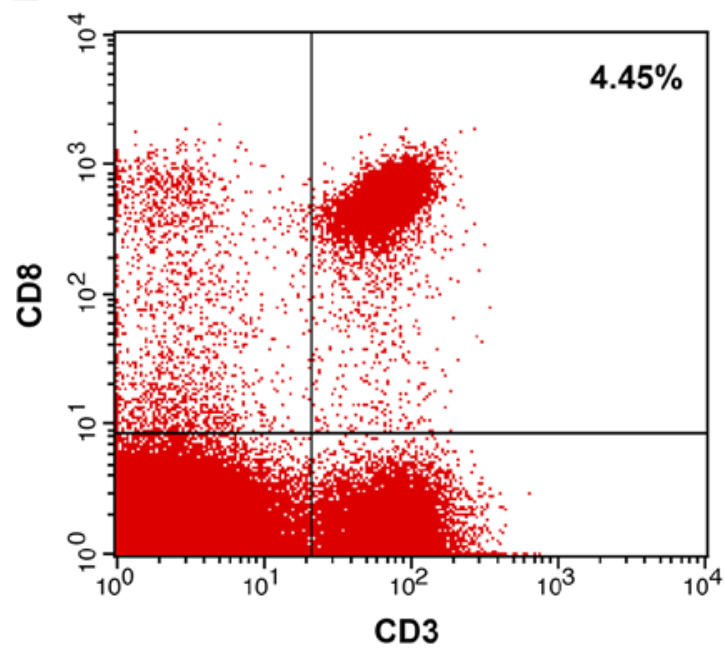

B

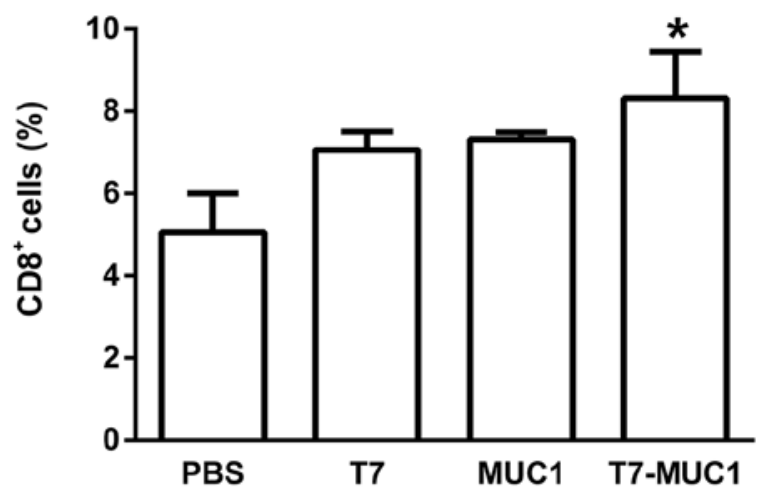

D

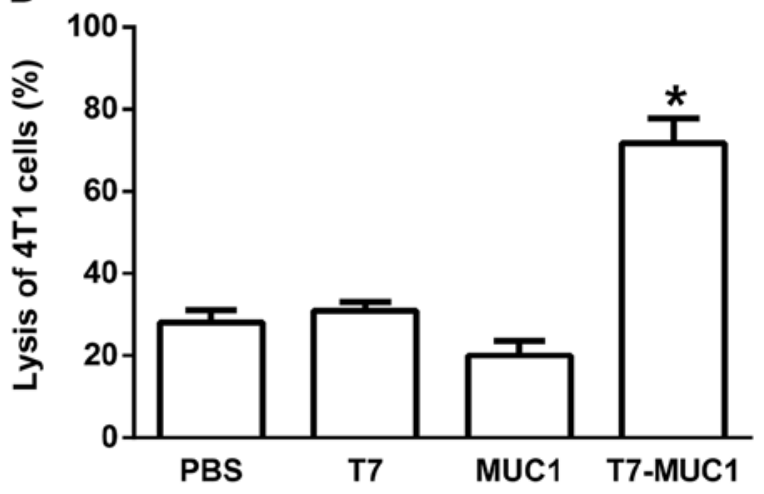

$\mathrm{F}$

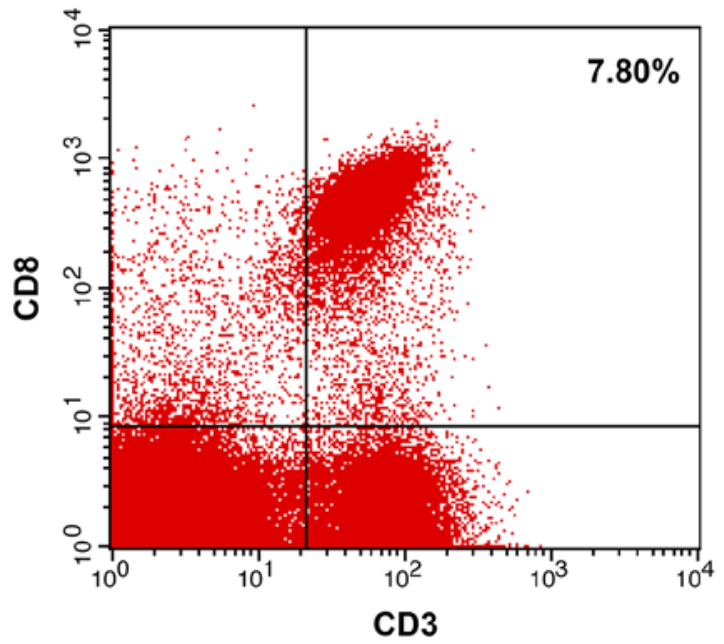

Figure 5. T7-MUC1 conjugate induces tumor-specific immune responses. (A and B) Percentages of (A) $\mathrm{CD}^{+} / \mathrm{CD}^{+} \mathrm{T}$ cells and $(\mathrm{B}) \mathrm{CD}^{+} / \mathrm{CD} 3^{+} \mathrm{T}$ cells in the total splenocytes were measured using flow cytometry. (C) Induction of cytolytic T cells in the tumor model mice was measured using a CTL assay. (D) Induction of ADCC in the tumor model mice was measured using an ADCC assay. (E and F) Representative flow cytometry results of CD8 ${ }^{+} / \mathrm{CD}^{+} \mathrm{T}$ cells in splenocytes in the (E) PBS and (F) T7-MUC1 groups. Data are presented as the mean \pm SD; n=8. ${ }^{*} \mathrm{P}<0.05$ vs. PBS. MUC1, mucin 1; T7, Toll-like receptor 7 agonist; NK, natural killer; ADCC, antibody-dependent cell-mediated cytotoxicity; CTL, cytotoxic T lymphocytes.

TLR9 agonist, may be a potent adjuvant to a synthetic long peptide containing the human papilloma virus $16 \mathrm{E} 7$ antigen, which can induce multifunctional antigen specific $\mathrm{CD}^{+} \mathrm{T}$ cells with prophylactic and therapeutic benefits in a murine lung tumor model. Previous studies have also demonstrated that the conjugation of a TLR ligand and an antigen induces improved immunogenicity compared with a simple mixture of them, which is due to the fact that the distribution and metabolism of
TLR ligand and antigen may be more systemically coincident in the design of a conjugate $(8,18)$. Our previous study revealed the necessity of a covalent attachment in the design of tumor vaccines containing $\mathrm{T} 7$ and tumor-associated antigens $(12,16)$. Therefore, in the present study, a similar strategy was used to construct the T7-MUC1 vaccine by conjugating T7 to lysine and linking it to the N-terminal of MUC1 peptide at a ratio of $3: 1$. 
The results of the present study suggested that the T7-MUC1 conjugate caused a rapid induction of inflammatory mediators TNF- $\alpha$, IFN- $\gamma$ and IL-12 in mouse BMDCs and spleen lymphocytes in vitro, indicating the activation of innate immunity. As TNF- $\alpha$, IFN- $\gamma$ and IL-12 are Th1 cytokines (30), these results suggested that TLR7 activation may lead to Th1-biased immune responses. Furthermore, TLR-produced cytokines stimulate the expression of a number of co-stimulatory molecules, promoting the optimal performance of T and B cells (8). Additionally, $\operatorname{IgG}$ and $\mathrm{IgM}$ are two types of Ig produced by B cells based on a specific antigen, thus mediating complement-dependent cytotoxicity and ADCC activity (31). The results of the present study demonstrated that the T7-MUC1 vaccine elicited anti-MUC1 IgG and IgM antibodies in the immunized mice and was more effective compared with T7 or MUC1 alone. Therefore, the present results indicated that the covalent attachment of $\mathrm{T} 7$ facilitated selective internalization by TLR7-expressing immune cells, such as B cells and APCs.

Following mouse immunization with the vaccines, the present study generated a tumor challenge model in BALB/c mice via subcutaneous implantation of mouse 4T1 breast cancer cells in order to investigate the in vivo antitumor effects of T7-MUC1. The results demonstrated that the T7-MUC1 conjugate exhibited the highest prophylactic performance among the tested vaccines for tumor treatment, and several aspects of tumor-specific immune responses were further examined. The results indicated that significant cytotoxicity to 4T1 tumor cells was caused by the antisera collected from mice immunized with T7-MUC1 with the presence of NK cells. In addition, the results of ADCC activity were similar to those of the IgG and IgM titers, suggesting the activation on humoral immunity by the T7-MUC1 vaccine. In terms of cellular immunity, CTL activity was identified to exhibit similar outcomes of tumor cell lysis as ADCC activity. Among the subsets of $\mathrm{T}$ cells, $\mathrm{CD} 4^{+}$and $\mathrm{CD} 8^{+} \mathrm{T}$ cells serve essential roles in the host defense against different types of cancer, such as melanoma (32). Naïve $\mathrm{CD}^{+} \mathrm{T}$ cells can differentiate into a variety of subsets, including Th1, Th2, Th9, Th17 and T regulatory cells, whereas naïve $\mathrm{CD}^{+} \mathrm{T}$ cells differentiate into memory and effector $\mathrm{CD}^{+} \mathrm{T}$ cells (33). In line with the CTL results, T7-MUC1 increased the proportions of $\mathrm{CD}^{+} / \mathrm{CD}^{+} \mathrm{T}$ cells, but not $\mathrm{CD}^{+} / \mathrm{CD}^{+} \mathrm{T}$-cells, in splenocytes, which was expected as CTLs belong to the CD8 ${ }^{+}$ T-cell subset. Therefore, the present results may facilitate the development of a MUC1-derived vaccine, which may initiate a response against residual cancer cells, thus improving long-term survival.

DC-CIK adoptive cellular immunotherapy is significant due to its strong antitumor activity against a broad spectrum of solid tumor types, including breast cancer, as DCs are stimulators of tumor-specific T cell responses, and CIKs are ex vivo-expanded $\mathrm{T}$ lymphocytes with a NK/T-cell phenotype $(34,35)$. The present study used an ex vivo model to examine the antitumor mechanisms of T7-MUC1; DCs were treated with the vaccines, followed by co-culture of antigen-loaded DCs and CIKs, and their toxicity to tumor cells was tested using the LDH method. The present results demonstrated that T7-MUC1 significantly induced the DC-CIK response in human breast cancer MCF-7 cells with high MUC1 expression, but not in those with low (K562) or no (MB231) MUC1 expression. The results also demonstrated that the antigen was more effective when added on day 3 compared with day 5 . Collectively, the results of the present study demonstrated the selectivity and specificity of T7-MUC1 for the application of cellular immunotherapy in breast cancer.

In conclusion, the novel $\mathrm{T} 7$ was conjugated with the unglycosylated MUC1 peptide to develop a safe and effective immunotherapeutic vaccine against breast cancer. The results of the present study demonstrated that $\mathrm{T} 7$ elicited non-specific immune responses and strengthened the specific humoral and cellular immune responses to the MUC1 antigen. In addition, the vaccine induced CTLs and ADCC-mediating antibodies recognizing MUC1, and overall exhibited a potential to inhibit tumor growth. Therefore, this vaccine candidate may have beneficial effects for the prevention of tumor recurrence in patients with breast cancer.

\section{Acknowledgements}

Not applicable.

\section{Funding}

The present study was supported by the Guangdong Science and Technology Department (grant nos. 2017A030310400 and 2018A0303130225) and the Shenzhen Science and Technology Innovation Commission (grant nos. JCYJ20180305163318492 and JSGG20160331161046511).

\section{Availability of data and materials}

The datasets used and/or analyzed during the current study are available from the corresponding author on reasonable request.

\section{Authors' contributions}

XW, GJ, YL and LT designed the study. YL, LT, NG, YWD and JZ performed the experiments. YL, LT, NG, YQD and ZW analyzed the data. XW, GJ, YL and LT wrote the manuscript. All authors read and approved the final manuscript.

\section{Ethics approval and consent to participate}

Ethics approval was obtained for the use of human tissues in the present study by the Medical Ethics Committee of the Third Affiliated Hospital of Shenzhen University (approval no. 2019-SZLH-LW-009), and informed consent was provided by the healthy donors. The protocols of animal experiments were approved by the Laboratory Animal Ethics Committee of Shenzhen University (approval no. AEWC-201712025).

\section{Patient consent for publication}

Not applicable.

\section{Competing interests}

The authors declare that they have no competing interests. 


\section{References}

1. Curigliano G, Spitaleri G, Pietri E, Rescigno M, de Braud F Cardillo A, Munzone E, Rocca A, Bonizzi G, Brichard V, et al: Breast cancer vaccines: A clinical reality or fairy tale? Ann Oncol 17: 750-762, 2006.

2. Sangha R and Butts C: L-BLP25: A peptide vaccine strategy in non small cell lung cancer. Clin Cancer Res 13 (Suppl): S4652-S4654, 2007.

3. Ninkovic T and Hanisch FG: O-glycosylated human MUC1 repeats are processed in vitro by immunoproteasomes J Immunol 179: 2380-2388, 2007.

4. Roulois D, Grégoire M and Fonteneau JF: MUC1-specific cytotoxic T lymphocytes in cancer therapy: Induction and challenge. Biomed Res Int 2013: 871936, 2013.

5. Taylor-Papadimitriou J, Burchell JM, Graham R and Beatson R: Latest developments in MUC1 immunotherapy. Biochem Soc Trans 46: 659-668, 2018.

6. Scheid E, Major P, Bergeron A, Finn OJ, Salter RD, Eady R, Yassine-Diab B, Favre D, Peretz Y, Landry C, et al: Tn-MUC1 DC vaccination of rhesus macaques and a phase I/II trial in patients with nonmetastatic castrate-resistant prostate cancer. Cancer Immunol Res 4: 881-892, 2016.

7. Tosch C, Bastien B, Barraud L, Grellier B, Nourtier V, Gantzer M, Limacher JM, Quemeneur E, Bendjama K and Préville X: Viral based vaccine TG4010 induces broadening of specific immune response and improves outcome in advanced NSCLC. J Immunother Cancer 5: 70, 2017.

8. Lakshminarayanan V, Thompson P, Wolfert MA, Buskas T, Bradley JM, Pathangey LB, Madsen CS, Cohen PA, Gendler SJ and Boons GJ: Immune recognition of tumor-associated mucin MUC1 is achieved by a fully synthetic aberrantly glycosylated MUC1 tripartite vaccine. Proc Natl Acad Sci USA 109: 261-266, 2012.

9. Braunstein MJ, Kucharczyk J and Adams S: Targeting toll-like receptors for cancer therapy. Target Oncol 13: 583-598, 2018.

10. Zhao S, Gao N, Qi H, Chi H, Liu B, He B, Wang J, Jin Z, He X, Zheng H, et al: Suppressive effects of sunitinib on a TLR activation-induced cytokine storm. Eur J Pharmacol 854: 347-353, 2019.

11. Coffman RL, Sher A and Seder RA: Vaccine adjuvants: Putting innate immunity to work. Immunity 33: 492-503, 2010.

12. Wang XD, Gao NN, Diao YW, Liu Y, Gao D, Li W, Wan YY, Zhong JJ and Jin GY: Conjugation of toll-like receptor-7 agonist to gastric cancer antigen MG7-Ag exerts antitumor effects. World J Gastroenterol 21: 8052-8060, 2015.

13. Vascotto F, Petschenka J, Walzer KC, Vormehr M, Brkic M, Strobl S, Rosemann R, Diken M, Kreiter S, Töreci Ö and Sahin U: Intravenous delivery of the toll-like receptor 7 agonist $\mathrm{SC} 1$ confers tumor control by inducing a $\mathrm{CD} 8^{+} \mathrm{T}$ cell response. Oncoimmunology 8: 1601480, 2019.

14. Kim H, Sehgal D, Kucaba TA, Ferguson DM, Griffith TS and Panyam J: Acidic pH-responsive polymer nanoparticles as a TLR7/8 agonist delivery platform for cancer immunotherapy. Nanoscale 10: 20851-20862, 2018.

15. Gao D, Liu Y, Diao Y, Gao N, Wang Z, Jiang W and Jin G: Synthesis and evaluation of conjugates of novel TLR7 inert ligands as self-adjuvanting immunopotentiators. ACS Med Chem Lett 6: 249-253, 2015.

16. Wang X, Liu Y, Diao Y, Gao N, Wan Y, Zhong J, Zheng H, Wang $Z$ and Jin G: Gastric cancer vaccines synthesized using a TLR7 agonist and their synergistic antitumor effects with 5-fluorouracil. J Transl Med 16: 120, 2018.

17. Zhu J, He S, Du J, Wang Z, Li W, Chen X, Jiang W, Zheng D and Jin G: Local administration of a novel Toll-like receptor 7 agonist in combination with doxorubicin induces durable tumouricidal effects in a murine model of $\mathrm{T}$ cell lymphoma. J Hematol Oncol 8: 21, 2015

18. Lin G, Wang X, Yi W, Zhang C, Xu G, Zhu X, Cai Z, Liu Y, Diao Y, Lin MC and Jin G: A conjugate of octamer-binding transcription factor 4 and toll-like receptor 7 agonist prevents the growth and metastasis of testis embryonic carcinoma. J Transl Med 13: 166, 2015.
19. Qiao G, Wang X, Zhou L, Zhou X, Song Y, Wang S, Zhao L, Morse MA, Hobeika A, Song J, et al: Autologous dendritic cell-cytokine induced killer cell immunotherapy combined with S-1 plus cisplatin in patients with advanced gastric cancer: A prospective study. Clin Cancer Res 25: 1494-1504, 2019.

20. Akram M, Iqbal M, Daniyal M and Khan AU: Awareness and current knowledge of breast cancer. Biol Res 50: 33, 2017.

21. Yang Y: Cancer immunotherapy: Harnessing the immune system to battle cancer. J Clin Invest 125: 3335-3337, 2015.

22. Mohit E, Hashemi A and Allahyari M: Breast cancer immunotherapy: Monoclonal antibodies and peptide-based vaccines. Expert Rev Clin Immunol 10: 927-961, 2014.

23. Morse MA and Whelan M: A year of successful cancer vaccines points to a path forward. Curr Opin Mol Ther 12: 11-13, 2010.

24. Cazet A, Julien S, Bobowski M, Burchell J and Delannoy P: Tumour-associated carbohydrate antigens in breast cancer. Breast Cancer Res 12: 204, 2010.

25. Beatson RE, Taylor-Papadimitriou $\mathrm{J}$ and Burchell JM: MUC immunotherapy. Immunotherapy 2: 305-327, 2010.

26. Adluri S, Gilewski T, Zhang S, Ramnath V, Ragupathi G and Livingston P: Specificity analysis of sera from breast cancer patients vaccinated with MUC1-KLH plus QS-21. Br J Cancer 79: 1806-1812, 1999.

27. Musselli C, Ragupathi G, Gilewski T, Panageas KS, Spinat Y and Livingston PO: Reevaluation of the cellular immune response in breast cancer patients vaccinated with MUC1. Int J Cancer 97: 660-667, 2002.

28. Khan A, Bakhru P, Saikolappan S, Das K, Soudani E, Singh CR, Estrella JL, Zhang D, Pasare C, Ma Y, et al: An autophagy-inducing and TLR-2 activating BCG vaccine induces a robust protection against tuberculosis in mice. NPJ Vaccines 4: 34, 2019.

29. Maynard SK, Marshall JD, MacGill RS, Yu L, Cann JA, Cheng LI, McCarthy MP, Cayatte C and Robbins SH: Vaccination with synthetic long peptide formulated with $\mathrm{CpG}$ in an oil-in-water emulsion induces robust E7-specific CD8 T cell responses and TC-1 tumor eradication. BMC Cancer 19: 540, 2019.

30. Habijanic J, Berovic M, Boh B, Plankl M and Wraber B: Submerged cultivation of Ganoderma lucidum and the effects of its polysaccharides on the production of human cytokines TNF- $\alpha$, IL-12, IFN- $\gamma$, IL-2, IL-4, IL-10 and IL-17. N Biotechnol 32: 85-95, 2015.

31. Sánchez Ramírez J, Morera Díaz Y, Bequet-Romero M, Hernandez-Bernal F, Selman-Housein Bernal KH, de la Torre Santos A, Santiesteban Álvarez ER, Martín Bauta Y, Bermudez Badell $\mathrm{CH}$, de la Torre Pupo J, et al: Characteristics of the specific humoral response in patients with advanced solid tumors after active immunotherapy with a VEGF vaccine, at different antigen doses and using two distinct adjuvants. BMC Immunol 18: 39, 2017.

32. Mei Y, Zhao L, Liu Y, Gong H, Song Y, Lei L, Zhu Y, Jin Z, Ma S, Hu B, et al: Combining DNA vaccine and AIDA-1 in attenuated salmonella activates tumor-specific CD4 ${ }^{+}$and $\mathrm{CD} 8^{+}$ T-cell responses. Cancer Immunol Res 5: 503-514, 2017.

33. Jiang $\mathrm{S}$ and Yan W: T-cell immunometabolism against cancer. Cancer Lett 382: 255-258, 2016.

34. Lin M, Liang S, Jiang F, Xu J, Zhu W, Qian W, Hu Y, Zhou Z, Chen J, Niu L, et al: 2003-2013, a valuable study: Autologous tumor lysate-pulsed dendritic cell immunotherapy with cytokine-induced killer cells improves survival in stage IV breast cancer. Immunol Lett 183: 37-43, 2017.

35. Zhao Y,QiaoG,Wang X,Song Y,Zhou X,Jiang N,Zhou L,Huang H, Zhao J, Morse MA, et al: Combination of DC/CIK adoptive T cell immunotherapy with chemotherapy in advanced non-small-cell lung cancer (NSCLC) patients: A prospective patients' preference-based study (PPPS). Clin Transl Oncol 21: 721-728: 2019.

This work is licensed under a Creative Commons Attribution-NonCommercial-NoDerivatives 4.0 International (CC BY-NC-ND 4.0) License. 